\title{
Human NCT
}

\begin{tabular}{|c|c|c|c|c|}
\hline $\begin{array}{c}\text { Fragment } \\
(\#)\end{array}$ & \begin{tabular}{|c|}
$\begin{array}{c}\text { Peptide } \\
\text { (a.a.residues) }\end{array}$ \\
\end{tabular} & $\begin{array}{c}{[\mathbf{M}+\mathbf{H}]} \\
(\mathbf{D a})\end{array}$ & $\begin{array}{c}{[\mathrm{M}+2 \mathrm{H}]} \\
(\mathbf{D a}) \\
\end{array}$ & $\begin{array}{c}\text { Observed Mass } \\
\text { (Da) }\end{array}$ \\
\hline T1 & $1-15$ & 1291.57 & 646.29 & 654.34 (Oxidation) $\left(2^{+}\right)$ \\
\hline $\mathrm{T} 2$ & 16-19 & 458.31 & 229.66 & \\
\hline T3 & $20-32$ & 1407.79 & 704.40 & \\
\hline $\mathrm{T} 4$ & $33-38$ & 661.33 & 331.17 & \\
\hline T5 & $39-39$ & 147.11 & 74.06 & \\
\hline T6 & $40-46$ & 860.52 & 430.77 & $430.78\left(2^{+}\right)$ \\
\hline $\mathrm{T} 7$ & $47-52$ & 646.33 & 323.67 & \\
\hline $\mathrm{T} 8$ & $53-78$ & 2706.39 & 1353.70 & \\
\hline T9 & $79-101$ & 2676.31 & 1337.16 & $891.78\left(3^{+}\right)$ \\
\hline $\mathrm{T} 10$ & $102-105$ & 560.29 & 280.65 & \\
\hline T11 & $106-110$ & 635.31 & 318.16 & \\
\hline $\mathrm{T} 12$ & $111-112$ & 260.20 & 130.60 & \\
\hline $\mathrm{T} 13$ & $113-114$ & 232.14 & 116.57 & \\
\hline T14 & $115-117$ & 363.20 & 182.10 & \\
\hline $\mathrm{T} 15$ & $118-160$ & 4415.09 & 2208.05 & $1473.25\left(3^{+}\right)$ \\
\hline T16 & $161-190$ & 3518.66 & 1759.84 & \\
\hline $\mathrm{T} 17$ & 191-193 & 359.27 & 180.14 & \\
\hline T18 & $194-232$ & 4338.94 & 2169.98 & \\
\hline T19 & $232-233$ & 175.12 & 88.06 & \\
\hline $\mathrm{T} 20$ & $234-274$ & 4582.25 & 2291.63 & \\
\hline $\mathrm{T} 21$ & $275-281$ & 715.45 & 358.23 & \\
\hline $\mathrm{T} 22$ & $282-285$ & 490.26 & 245.64 & \\
\hline $\mathrm{T} 23$ & $286-314$ & 3010.53 & 1005.77 & $1004.19\left(2^{+}\right)$ \\
\hline $\mathrm{T} 24$ & $315-323$ & 969.54 & 485.27 & $485.27\left(2^{+}\right)$ \\
\hline $\mathrm{T} 25$ & $324-342$ & 2244.04 & 1122.53 & $749.27\left(3^{+}\right)$ \\
\hline $\mathrm{T} 26$ & $343-349$ & 915.40 & 458.20 & $\begin{array}{c}458.22 / 474.20(2 \text { Oxidation }) \\
\left(2^{+}\right)\end{array}$ \\
\hline $\begin{array}{c}\text { T27 } \\
\text { T27-T28 }\end{array}$ & $\begin{array}{l}350-351 \\
350-371 \\
\end{array}$ & $\begin{array}{c}204.13 \\
2244.31\end{array}$ & 102.57 & $1223.18\left(2^{+}\right) / 815.76\left(3^{+}\right)$ \\
\hline T28 & $352-371$ & 2260.20 & 1130.60 & $1130.59\left(2^{+}\right) / 754.06\left(3^{+}\right)$ \\
\hline T29 & $372-386$ & 1771.87 & 886.44 & 591.30/596.62 (Oxidation) $\left(3^{+}\right)$ \\
\hline $\mathrm{T} 30$ & $387-391$ & 604.31 & 302.66 & \\
\hline T31 & $392-403$ & 1372.73 & 686.87 & $686.88\left(2^{+}\right) / 458.25\left(3^{+}\right)$ \\
\hline T32 & $404-414$ & 1039.63 & 520.32 & $520.33\left(2^{+}\right)$ \\
\hline T33 & $415-429$ & 1704.91 & 852.96 & $568.98\left(3^{+}\right)$ \\
\hline T34 & $430-432$ & 435.27 & 218.14 & \\
\hline T35 & $433-434$ & 246.16 & 123.58 & \\
\hline T36 & $435-451$ & 1765.90 & 883.45 & \\
\hline
\end{tabular}




\begin{tabular}{|c|c|c|c|c|}
\hline T37 & $452-485$ & 4014.84 & 2007.93 & $543.33\left(2^{+}\right)$ \\
\hline T38 & $486-496$ & 1085.63 & 543.32 & $483.81\left(2^{+}\right)$ \\
\hline T39 & $497-520$ & 2554.24 & 1227.63 & $761.74\left(3^{+}\right)$ \\
\hline T40 & $521-528$ & 966.60 & 483.81 & \\
T40-T41 & $521-539$ & 2284.71 & 1142.86 & \\
\hline T41 & $529-539$ & 1336.49 & 668.75 & $549.25\left(3^{+}\right)$ \\
\hline T42 & $540-543$ & 531.29 & 266.15 & \\
\hline T43 & $544-583$ & 4297.21 & 2149.11 & \\
\hline T44 & $584-591$ & 934.39 & 467.11 & \\
T44-T45 & $584-597$ & 1644.73 & & \\
\hline T45 & $592-597$ & 673.35 & 337.18 & \\
\hline T46 & $598-616$ & 2309.05 & 1155.03 & \\
\hline T47 & $617-619$ & 385.26 & 193.13 & \\
\hline T48 & $620-622$ & 377.20 & 189.10 & \\
\hline T49 & $623-626$ & 434.24 & 217.62 & \\
\hline T50 & $627-629$ & 359.24 & 180.12 & \\
\hline T51 & $630-652$ & 2663.23 & 1332.12 & $1332.13\left(2^{+}\right) / 888.41\left(3^{+}\right)$ \\
\hline T52 & $653-654$ & 333.19 & 167.10 & \\
\hline T53 & $655-657$ & 403.23 & 202.12 & \\
\hline T54 & $658-659$ & 246.16 & 123.58 & \\
\hline T55 & $660-666$ & 791.50 & 396.26 & \\
\hline T56 & $667-693$ & 2983.68 & 1492.35 & \\
\hline T57 & $694-702$ & 1001.58 & 501.29 & \\
\hline T58 & $703-709$ & 722.34 & 361.67 & \\
\hline & & & & \\
\hline
\end{tabular}




\section{Human PEN-2}

\begin{tabular}{|c|c|c|c|c|}
\hline $\begin{array}{c}\text { Fragment } \\
(\#)\end{array}$ & $\begin{array}{c}\text { Peptide } \\
\text { (a.a.residues) }\end{array}$ & $\begin{array}{c}{[\mathbf{M}+\mathbf{H}]} \\
\text { (Da) }\end{array}$ & $\begin{array}{c}{[\mathbf{M}+\mathbf{2 H}]} \\
(\mathbf{D a})\end{array}$ & $\begin{array}{c}\text { Observed Mass } \\
\text { (Da) }\end{array}$ \\
\hline & & & & \\
\hline $\mathrm{T} 1$ & $1-5$ & 662.33 & 331.67 & \\
\hline $\mathrm{T} 2$ & $6-11$ & 705.34 & 353.17 & \\
\hline T3 & $12-16$ & 618.34 & 309.67 & \\
\hline T4 & $17-17$ & 147.11 & 74.06 & \\
\hline T5 & $18-39$ & 2816.48 & 1408.74 & \\
\hline T6 & $40-54$ & 1723.89 & 862.45 & $862.42\left(2^{+}\right)$ \\
\hline T7 & $55-59$ & 680.35 & 340.68 & \\
\hline T8 & $60-84$ & 3012.69 & 1506.85 & \\
\hline T9 & $85-101$ & 1807.93 & 904.47 & $904.44\left(2^{+}\right)$ \\
\hline
\end{tabular}




\section{Human Aph1a2}

\begin{tabular}{|c|c|c|c|c|}
\hline $\begin{array}{c}\text { Fragment } \\
\text { (\#) }\end{array}$ & $\begin{array}{c}\text { Peptide } \\
\text { (a.a.residues) }\end{array}$ & $\begin{array}{c}{[\mathbf{M}+\mathbf{H}]} \\
\text { (Da) }\end{array}$ & $\begin{array}{c}{[\mathbf{M}+\mathbf{2 H}]} \\
\text { (Da) }\end{array}$ & $\begin{array}{c}\text { Observed Mass } \\
\text { (Da) }\end{array}$ \\
\hline T1 & $1-31$ & 3206.66 & 1603.83 & \\
\hline T2 & $32-62$ & 3497.05 & 1749.03 & \\
\hline T3 & $63-66$ & 448.22 & 224.61 & \\
\hline T4 & $67-87$ & 2336.34 & 1168.67 & \\
\hline T5 & $88-92$ & 691.35 & 346.18 & \\
\hline T6 & $93-95$ & 373.28 & 187.14 & \\
\hline T7 & $96-96$ & 147.11 & 74.06 & \\
\hline T8 & $97-109$ & 1319.61 & 660.31 & $660.30\left(2^{+}\right)$ \\
\hline T9 & $110-115$ & 672.40 & 336.71 & $336.70\left(2^{+}\right)$ \\
\hline T10 & $116-184$ & 7353.79 & 3677.40 & \\
\hline T11 & $185-185$ & 175.12 & 88.06 & \\
\hline T12 & $186-186$ & 175.12 & 88.06 & \\
\hline T13 & $187-237$ & 5511.92 & 2756.47 & \\
\hline T14 & $238-241$ & 503.29 & 252.15 & \\
T14-T15 & $238-246$ & 1075.60 & 538.31 & $538.32\left(2^{+}\right)$ \\
\hline T15 & $242-246$ & 591.33 & 296.17 & \\
\hline T16 & $247-247$ & 175.12 & 88.06 & \\
\hline T17 & $248-252$ & 634.28 & 317.64 & \\
\hline T18 & $253-260$ & 938.51 & 469.76 & $469.74\left(2^{+}\right)$ \\
\hline T19 & $261-265$ & 570.28 & 285.64 & \\
\hline & & & & \\
\hline
\end{tabular}




\section{Human PS1}

\begin{tabular}{|c|c|c|c|c|}
\hline $\begin{array}{l}\text { Fragment } \\
\text { (\#) }\end{array}$ & $\begin{array}{c}\text { Peptide } \\
\text { (a.a.residues) }\end{array}$ & $\begin{array}{c}{[\mathbf{M}+\mathbf{H}]} \\
(\mathbf{D a})\end{array}$ & $\begin{array}{c}{[\mathbf{M}+\mathbf{2 H}]} \\
(\mathbf{D a})\end{array}$ & $\begin{array}{c}\text { Observed Mass } \\
\text { (Da) }\end{array}$ \\
\hline T1 & $1-27$ & 3093.44 & 1547.22 & \\
\hline $\mathrm{T} 2$ & $28-33$ & 733.32 & 367.17 & \\
\hline T3 & $34-35$ & 304.16 & 152.58 & \\
\hline T4 & $36-41$ & 798.35 & 399.68 & \\
\hline $\mathrm{T} 4-\mathrm{T} 5$ & $36-42$ & & & $477.24\left(2^{+}\right)$ \\
\hline $\mathrm{T} 5$ & $42-42$ & 175.12 & 88.06 & \\
\hline T6 & $43-60$ & 1902.95 & 951.98 & \\
\hline $\mathrm{T} 7$ & $61-76$ & 1932.89 & 966.95 & $966.91\left(2^{+}\right)$ \\
\hline $\mathrm{T} 8$ & $77-80$ & 438.24 & 219.62 & \\
\hline T9 & $81-101$ & 2312.33 & 1156.67 & \\
\hline T10 & $102-108$ & 859.43 & 430.22 & \\
\hline T11 & 109-109 & 147.11 & 74.06 & \\
\hline $\mathrm{T} 11-\mathrm{T} 12$ & $109-128$ & & & $766.68\left(3^{+}\right)$ \\
\hline T12 & $110-128$ & 2170.03 & 1085.53 & $723.98\left(3^{+}\right)$ \\
\hline T13 & $129-155$ & 2937.77 & 1469.39 & \\
\hline T14 & $156-157$ & 338.18 & 169.60 & \\
\hline T15 & $158-160$ & 413.19 & 207.10 & \\
\hline T16 & $161-187$ & 3215.83 & 1608.42 & \\
\hline T17 & $188-216$ & 3322.77 & 1661.89 & \\
\hline T18 & $217-220$ & 442.28 & 221.64 & \\
\hline T19 & $221-239$ & 2166.24 & 1083.63 & \\
\hline T20 & $240-265$ & 2975.64 & 1488.32 & \\
\hline T21 & $266-269$ & 442.28 & 221.64 & \\
\hline T21-T22 & 266-278 & 1498.79 & & $500.58\left(3^{+}\right)$ \\
\hline T22 & $270-278$ & 1077.25 & 538.77 & $\begin{array}{c}538.75 / 546.74(\text { Oxidation) } \\
\left(2^{+}\right)\end{array}$ \\
\hline T23 & $279-307$ & 3282.58 & 1641.79 & \\
\hline T24 & $308-308$ & 175.12 & 88.06 & \\
\hline $\mathrm{T} 25$ & 309-311 & 33.21 & 167.11 & \\
\hline T26 & $312-314$ & 348.19 & 174.60 & \\
\hline $\mathrm{T} 27$ & $315-322$ & 969.43 & 485.22 & $485.26\left(2^{+}\right)$ \\
\hline $\mathrm{T} 28$ & $323-344$ & 2499.02 & 1250.01 & \\
\hline T29 & $345-352$ & 918.45 & 459.73 & $459.75\left(2^{+}\right)$ \\
\hline $\mathrm{T} 30$ & $353-358$ & 676.33 & 338.67 & \\
\hline T31 & $359-377$ & 2000.98 & 1000.99 & \\
\hline T32 & $378-380$ & 303.20 & 152.11 & \\
\hline T33 & $381-395$ & 1627.91 & 814.46 & $814.44\left(2^{+}\right)$ \\
\hline T34 & $396-429$ & 3523.93 & 1762.47 & \\
\hline T35 & $430-430$ & 147.11 & 74.06 & \\
\hline T36 & $431-467$ & 4297.21 & 2149.11 & \\
\hline
\end{tabular}


Fig. Sup.1. Mass spectral identification of $\gamma$-secretase-associated proteins. Nicastrin (NCBI accession numbers 11037796 (Mus Musculus, for purification in $\gamma$-30 cells) and 24638433 (Homo Sapiens, for purification in S-1 cells)), PS1-NTF, PS1-CTF (4506163), Aph1 (7705787) and Pen-2 (28144920) are the predominant proteins detected on the gels stained with silver nitrate (Figs. 1C and Sup.2) and identified by mass spectrometry. The peptides recovered after trypsine proteolysis (Tn) were identified by LC/MS and LC/MS/MS. For each peptide (Tn), the observed mass $(\mathrm{Da})$ is consistent with the corresponding mass $(M+H)$ divided by the number of charges $\left(n^{+}\right)$. For each combined peptide $(\operatorname{Tn}-\operatorname{Tn}+1)$, the observed mass is consistent with the sum of the two individual masses $(\mathrm{Tn}+\mathrm{Tn}+1, \mathrm{M}+\mathrm{H})-$ 1Da (one proton) - 18Da (H2O) (corresponding to one peptide bond), and divided by the number of charges $\left(\mathrm{n}^{+}\right)$.

Fraering et al, Fig. Sup.1 


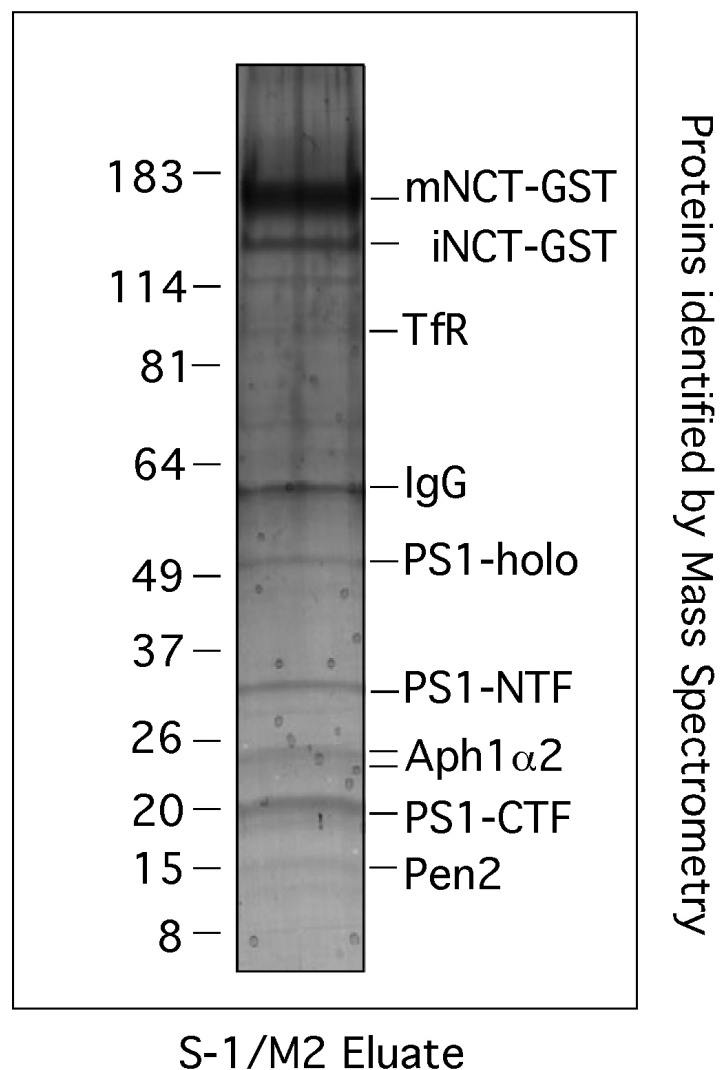

Fig. Sup.2. Purification of $\gamma$-secretase from $S-1$ cells and mass spectral identification of its associated proteins. The proteins recovered after the M2 anti-Flag affinity purification step (step 5, M2-Eluate, see Fig. 3A), obtained from $1.2 \times 10^{9} \mathrm{~S}-1$ cells, were pooled, concentrated by ultrafiltration using a Microcon 3KD centrifugation device, separated on a 4-20\% Tris-Glycine gel, and stained with silver nitrate. Each band visible on the gel was analyzed and the proteins identified by mass spectrometry, as described in Fig. 1C. Note the very similar silver stained protein profiles observed in the two purification procedures performed with $\gamma-30$ and S-1 cells (compare Figs Sup.2 and 1C).

Fraering et al, Fig. Sup.2 